\title{
De usuarios a clientes y de bibliotecarios a prescriptores de la información
}

\author{
Victòria Rodrigo Fuentes \\ Biblioteca Central Xavier Amorós de Reus
}

\section{Resumen}

El objetivo de este trabajo es demostrar que el perfil profesional de los bibliotecarios ha iniciado un giro hacia una nueva realidad, que los dibuja como prescriptores y gestores de la información, es decir, como carácter híbrido, no estrictamente documental. Este cambio, empezó con la aparición de la tecnología informática y ahora viene marcado por las expectativas de los usuarios, que a la vez, se posicionan como clientes y orientan la biblioteca del futuro con nuevas demandas de información.

Todo ello, en un entorno de incertidumbre provocado por la crisis económica y por los cambios gubernamentales, que las últimas elecciones han comportado y que a la misma vez depositan en los profesionales la responsabilidad de defender y reforzar el posicionamiento de la biblioteca pública delante de la nueva realidad política.

¿Un nuevo perfil profesional será el punto de equilibrio entre la oferta y la demanda en la nueva realidad informacional que envuelve la biblioteca?

Para dar respuesta a está cuestión se han analizado encuestas realizadas a usuarios, a profesionales de las bibliotecas, se han examinado ofertas profesionales y requerimientos solicitados en el sector bibliotecario y al mismo tiempo se han recogido opiniones de diferentes personas con altas responsabilidades dentro del campo de la biblioteca y la educación, tanto en el entorno privado como en el público. Los resultados denotan un vacío de formación en competencias y habilidades transversales entre estos profesionales. Carencias que se manifiestan en ámbitos tales como: trabajo en equipo, resolución de conflictos, colaboraciones, atención al cliente y habilidades de comunicación.

\section{Palabras clave}

Usuarios, Clientes, Bibliotecarios, Prescriptores de la información, Perfil profesional, Mercado laboral, Planes de estudio, Formación continuada, Competencias, Habilidades, Comunicación.

From users to customers and librarians prescribing information
Abstract
The aim of this paper is to demonstrate that the professional profile of librarians has begun a shift to a new reality, which draw them as prescribers and managers of information, ie as hybrid nature, not strictly documentary. This change began with the advent of computer technology and now is marked by the expectations of users, which in turn, are positioned as clients and guide the library of the future with new demands for information.
All this in an environment of uncertainty caused by the economic crisis and by the government changes, that the last elections have performed, and at the same time deposited in the professional responsibility to uphold and strengthen the position of the public library before the new political reality.
A new professional profile will be the point of balance between supply and demand in the new informational reality that surrounds the library?
To answer this question we have analyzed surveys of users, library professionals have been examined professional offers and requirements requested in the library sector and 
at the same time have collected opinions from different people with high responsibilities in the field of the library and education, both in the private and the public. The results indicate a gap in skills training and learning abilities among these professionals. Gaps that occur in areas such as teamwork, conflict resolution, collaboration, customer service and communication skills.

\section{Keywords}

Users, Customers, Librarians, Prescribers of information, Professional profile, Labor market, Curriculum, Continuing Education, Skills, Abilities, Communication.

La biblioteca, y en especial la biblioteca pública, está inmersa desde hace algunos años en una constante transformación. Unos cambios que, en un principio, han sido provocados por la tecnología, en general, y por las TIC, en particular, un conjunto que ha modificado de forma significativa los procesos de gestión, de trabajo y de relación de nuestras bibliotecas.

Cuando estos cambios no están del todo asumidos, tal como explica Hernández (2010) en la conferencia "Inmigrantes, lectura y biblioteca pública", surge una nueva realidad: la formada por una masa de usuarios de procedencia heterogénea, de intereses y de hábitos muy diferentes.

Todo ello ha significado que la biblioteca pública se haya convertido en un destacado espacio de sociabilidad, que la aleja un poco más de la galaxia del libro impreso para sumergirse en un mar formado por unos usuarios con un amplio abanico de necesidades informativas, que esperan encontrar respuesta en la biblioteca y en sus profesionales.

Además, el período de crisis que nos ha tocado vivir también está dejando huella en muchas instituciones públicas y las bibliotecas no son una excepción. Esta situación obliga a los bibliotecarios a trabajar con recortes presupuestarios considerables, que comportarán a la larga la redefinición de sus servicios.

Por tanto, las bibliotecas públicas se encuentran frente a una etapa de incertidumbre determinada por la crisis económica y las dificultades que ésta genera. En el caso de Cataluña, el cambio de gobierno de la Generalitat y de muchos ayuntamientos provoca dudas ante las nuevas políticas que se seguirán en este ámbito; por tanto habrá que esperar para comprobar la importancia que las diferentes administraciones otorgan a los servicios bibliotecarios y, en definitiva, donde sitúan sus prioridades.

Esta investigación analiza los datos recogidos en diferentes encuestas y estudios de opinión:

- Encuesta realizada en año 2011 a usuarios de la Biblioteca Central de Reus. La muestra recoge las opiniones de usuarios activos e inactivos de diferentes edades, sexo, origen i nivel socio-cultural, sobre usos y expectativas en la biblioteca pública.

- Encuesta realizada en el año 2011 a diferentes profesionales de bibliotecas públicas de la provincia de Tarragona. 
- La encuesta Imagina la biblioteca pública del siglo XXI, que la Generalitat de Cataluña realizó en el año 2008 y en la que participaron usuarios, profesionales, agentes municipales y personalidades del mundo de la cultura y de la educación.

- Encuesta "Inmigrantes, lectura y biblioteca pública" de Hilario Hernández, presentada en la inauguración de la "6a Escola d'Hivern de la Biblioteca Pública” organizada en Barcelona por la Universidad de Barcelona en el año 2010.

- El estudio Prospectiva de una profesión en constante evolución de José A. Gómez, Hilario Hernández y José A. Merlo, expuesto en las pasadas XII Jornadas Españolas de Documentación EBLIDA-NAPLE Conference 2011, donde profesionales del sector bibliotecario reflexionan sobre su futuro profesional.

- Selección de ofertas de trabajo en bibliotecas, tanto en el sector público como en el privado, que han sido recogidas en diferentes medios (publicaciones oficiales, oficinas de empleo, empresas privadas...) y enviadas por el Colegio de Bibliotecarios de Cataluña a algunos de sus miembros durante los años $2010 \mathrm{y}$ 2011.

\section{Los usuarios tienen la palabra}

En ausencia de estudios fiables, seria arriesgado determinar el perfil de nuestros usuarios y usuarias, como también lo seria hacerlo con la tipología de los ciudadanos y ciudadanas que conforman nuestra sociedad y que pueden variar considerablemente según el entorno geográfico y sociocultural al que pertenecen. Por tanto, las realidades entre las diversas bibliotecas pueden ser muy diferentes.

Está combinación de personas que integran nuestra comunidad ha tomado su turno de palabra para decir como quieren la biblioteca del futuro, como ha quedado reflejado en el proceso participativo Imagina la biblioteca pública del segle XXI, una iniciativa llevada a cabo en 2009 por el Departament de Cultura i Mitjans de Comunicació de la Generalitat de Catalunya. Esta consulta está basada en unos sondeos dirigidos, en primera instancia, a un buen número de personalidades representativas de la sociedad civil y política de Cataluña, al mundo cultural y educativo y de los medios de comunicación, lo cual permite conocer las inquietudes de un sector de la población plenamente implicado en el desarrollo cultural del país.

Este trabajo ha puesto sobre la mesa nuevas y viejas ideas orientadas a la búsqueda constante de las necesidades cambiantes de la ciudadanía; necesidades que giran alrededor del cambio permanente, del trabajo con recursos y agentes confluentes y dinámicos. Una biblioteca pública que en la vertiente de la información, la cultura y la formación tiene un papel reconocido y consolidado en la Cataluña de principios del segundo milenio.

Lamentablemente las encuestas también revelan el desconocimiento que de la biblioteca pública aún tiene una parte significativa de la población, que perseverante, conserva aún viejos clichés sobre sus limitaciones tanto a nivel cualitativo como 
cuantitativo en materia de recursos humanos, tecnológicos, de fondos, de horarios, de equipamientos, etcétera.

En líneas generales los resultados del documento ${ }^{1}$ se centran en: la mejora de mecanismos en el Sistema de Lectura Pública. En una mayor reglamentación que permita precisar los marcos competenciales, a menudo demasiado complejos por el número de administraciones que están implicadas alrededor de la biblioteca pública (Ayuntamientos, Diputaciones, Autonomías, etcétera) y la creación de modelos orientativos en aspectos profesionales que permitan trabajar en condiciones de homogeneidad y estabilidad capaces de "salvar" la biblioteca, en sus aspectos básicos, de los cambios políticos. Los resultados del estudio indican un doble objetivo en el camino que la biblioteca pública ha de seguir: por un lado, adecuar el sistema de lectura pública a las necesidades de la ciudadanía y, por otro, impulsar la organización del propio sistema de bibliotecas públicas.

En este sentido podemos afirmar que estos datos son, sin duda alguna, un fiel reflejo de las voluntades e inquietudes de las personas que participaron en el sondeo. Por eso cada administración tendría que tomar nota y evaluar las acciones propuestas para aplicarlas en el ejercicio de sus competencias, cuando estas se adecuen a las necesidades del Sistema de Lectura Pública. Siguiendo con esta iniciativa del Departament de Cultura de la Generalitat, el colectivo de la Biblioteca Central de Reus, preocupado por esta problemática, está recogiendo desde hace algún tiempo las demandas de información de los usuarios que se dirigen a sus mostradores, con una doble finalidad: la de conocer las necesidades de información de la comunidad a la que sirve y la de adaptar o diseñar sus servicios y sus fondos a estas demandas.

El análisis de los resultados constata un progresivo aumento de peticiones de productos en formato digital como son: los enlaces a recursos electrónicos; las fuentes de información, como textos legales; y los documentos electrónicos, como revistas.

También hay una considerable demanda de ayuda en la búsqueda de asesoramiento, dudas de funcionamiento o de orientación bibliográfica. Cuando un usuario solicita aclaraciones sobre una temática concreta, se responde a su solicitud explicándole las fuentes utilizadas y especificando el proceso de búsqueda. Así se realiza la formación del usuario al mismo tiempo que se le dan a conocer las diferentes herramientas de que dispone, motivándole a utilizarlas.

Los usuarios de servicios digitales, como el acceso al fondo histórico de la hemeroteca de la biblioteca han aumentado considerablemente, ya que el acceso, por vía Internet, al documento digitalizado, prevalece en detrimento de la posesión física de este. Sin embargo en la caso de los libros la mayoría de los usuarios prefieren acceder directamente a los volúmenes y evaluar así si responden o no a sus necesidades, antes que hacer la reserva online, ya que los catálogos bibliográficos virtuales todavía contienen, únicamente, datos básicos.

Se requiere pues, información de manera rápida, sencilla y con acceso desde Internet para hacerlo a cualquier hora y desde cualquier lugar. Se propone el acceso deslocalizado, es decir, el acceso desde diferentes puntos alternativos a la web, como 
blogs, Facebook, etcétera. Las necesidades de estos usuarios tienden hacia la autosuficiencia y la autogestión, y para ello necesitan interfaces como la que ofrece Google (una sola ventana y simplicidad).

\section{La biblioteca pública orientada a los usuarios}

En el caso de Cataluña podemos decir que la presencia de la biblioteca pública se está consolidando en los últimos años como resultado de la implicación que han demostrado las Administraciones Públicas en la destinación de recursos. De todas maneras, la creación de nuevos equipamientos, el impulso de políticas de fomento de la lectura y las mejoras de fondos bibliográficos, por sí mismos no son garantía de un buen servicio bibliotecario.

La biblioteca ha de ser útil a la comunidad a la que sirve y eso sólo se puede hacer con respuestas concretas para sus usuarios. A menudo se cuestionan los servicios que tradicionalmente ofrecen las bibliotecas: los usuarios no sólo ven la biblioteca como un espacio de lectura o de ocio, sino que aquellos que se dirigen a nuestros mostradores, desean que la información les sea entregada encapsulada, procesada, fácil de digerir. Es decir, esperan que el bibliotecario actúe como filtro cualitativo sobre la gran cantidad de información que recibe.

Seguramente ha llegado la hora de tener en cuenta que, para conseguir el éxito, es preciso que la biblioteca deje de considerar a los usuarios como conceptos abstractos y considerarlos como personas reales, con necesidades y deseos concretos, intentando establecer sinergias y complicidades.

Es el momento de introducir el término cliente en lugar del de usuario. Un concepto que hasta ahora se utilizaba sólo en el ámbito empresarial privado, pero que actualmente se ha expandido hacia los servicios públicos. Hasta hace poco, solo podíamos definir cliente como aquella persona que recibe un servicio y que paga por haberlo recibido, pero actualmente podemos considerar cliente todo aquel que recibe los beneficios de bienes y servicios, como son los estudiantes en los centros docentes o los pacientes en los centros hospitalarios. El ciudadano es, pues, un cliente de los servicios públicos porque ya los ha pagado de antemano con sus impuestos.

Las bibliotecas han de saber atraer y mantener a sus clientes. Una fórmula puede ser la de imitar la forma en que el sector comercial lo hace, sin renunciar ni a los principios, ni a los valores que la biblioteca tiene como servicio público. Un servicio orientado a la excelencia y a la eficacia, ya que una consideración diferente podría tener connotaciones negativas. Se ha de garantizar que la biblioteca conserve la consideración de beneficiosa para la comunidad y para los que la financian, que como he dicho, son los propios ciudadanos con sus tributos.

Por otro lado, el proyecto de una biblioteca pública no sólo ha de estar claramente orientada hacia los intereses de los usuarios, sino que precisa del apoyo del personal que trabaja en ella y del de las administraciones de las cuales depende. 


\section{Los bibliotecarios tienen la respuesta}

La respuesta de los profesionales de la biblioteca es fundamental para hacer que esta sea atractiva, accesible y capaz de asimilar las exigencias que día tras día les trasladan los usuarios.

De este modo hemos querido recoger la opinión que sobre el futuro de su profesión tienen algunos profesionales del entorno de la biblioteca pública del Camp de Tarragona. La mayoría de sus respuestas giran entorno a estos tres ejes principales:

- El papel de mediador entre usuario e información. Es decir, tendrán que hacer de filtro entre las amplias demandas de información y las fuentes de donde proceden.

- La tecnología seguirá marcando la evolución de la profesión. Para ofrecer mejores y más adecuados servicios a los usuarios será necesario utilizar las nuevas tecnologías y herramientas que van apareciendo en el mercado y que se adapten mejor a nuestras necesidades.

- La formación de usuarios autosuficientes se convertirá en una necesidad, más que en un objetivo, resultado de la nueva realidad informativa in crescendo y por los recortes presupuestarios que se avecinan. Estos recortes afectarán al servicio y a los empleados encargados de prestarlo, en situación de precariedad laboral, como las que están surgiendo a causa de la subcontratación de personal por parte de la administración pública.

En consecuencia es necesario reflexionar sobre el papel que estos profesionales han de desarrollar en el futuro, por lo que contemplaremos algunas de sus propuestas y inquietudes recogidas en el estudio Prospectiva de una profesión en constante evolución de Gómez-Hernández et al. (2011). Este documento recoge los resultados de una encuesta realizada a una muestra representativa de profesionales titulados del mundo de la biblioteca, del archivo y del museo. De este grupo hemos escogido las conclusiones sobre educación, formación y sobre el futuro de la profesión, directamente relacionadas con los bibliotecarios.

En el campo de la educación y la formación estos profesionales consideran adecuados los conocimientos académicos recibidos para su formación, pero reconocen que la constante innovación tecnológica ha cambiado su actividad profesional. Es evidente que la tecnología facilita su tarea, pero todos coinciden en la necesidad de una formación continuada para adaptarse a los cambios, así como para actualizar sus conocimientos.

Para el futuro prevén una mayor confluencia entre los profesionales de la información, es decir, archiveros, bibliotecarios y personal de museos. Los libros electrónicos irán aumentando su protagonismo en los fondos de nuestras bibliotecas, mientras que el número de documentos impresos se estabilizará, para acabar reduciendo su presencia paulatinamente. También están convencidos del aumento del número de recursos en línea, catálogos y otros servicios con elementos participativos. 


\section{Un nuevo perfil ${ }_{\mathbf{d}}$ Punto de equilibrio ente la oferta y la demanda?}

El mercado laboral también impone sus leyes en el entorno bibliotecario. Después de analizar las ofertas de trabajo, recogidas por el Colegio de Bibliotecarios de Cataluña y remitidas a sus colegiados, las conclusiones son las siguientes: los profesionales de las bibliotecas han de cumplir una serie de requisitos entre los que destacamos, ser expertos en el uso de las últimas tecnologías, saber trabajar en equipo, ser capaces de concebir y evaluar sistemas de acceso a la información y resolver las necesidades de los usuarios.

Aunque, según el artículo Prospectiva de una profesión en constante evolución citado anteriormente- los profesionales en activo valoran como satisfactoria su formación, hay que considerar un nuevo enfoque académico que vaya más allá de los contenidos referidos a la información y al conocimiento. Muchos profesionales denuncian una falta de conexión entre los planteamientos de la oferta y los requerimientos de la demanda, es decir entre la formación académica y las ofertas de trabajo. Esta cuestión ha estado largamente reivindicada por todos los agentes del sector, pero desafortunadamente no se acaba de solucionar.

Un hecho determinante es que los bibliotecarios al acabar sus estudios carecen de especialización, obtienen un título de carácter general, mientras que el mercado está pidiendo profesionales especializados en diferentes áreas: tecnológica, científica, humanista... Actualmente esta formación la obtienen los profesionales con el paso del tiempo y fruto de la experiencia en su puesto de trabajo, lo que supone la inversión de un tiempo considerable a nivel organizativo para formar a la persona implicada. Es lo que se llama formación continuada.

Tal como señala Atenas (2010): "si se observa la relación entre las ofertas de trabajo con los programas de formación profesional, aparece una necesidad clara de explicar a las universidades que han de disponer de programas específicos para preparar profesionales especializados con capacidades más transversales en todos los campos, ya que los profesionales han de poder entender y adaptarse a los ambientes laborales altamente tensionados, y que puedan desarrollarse eficientemente y sean considerados profesionales que puedan gestionar las capacidades más importantes, como la comunicación, la planificación y el presupuesto estratégico, habilidades de presentación, gerencia de proyectos, y con sentido del humor".

No obstante, el sistema universitario consciente del problema e inmerso en un escenario de crisis y reducción de número de alumnos, como el que nos presentó Frias y Villalba (2009) en las XV Jornadas bibliotecarias de Andalucía, ha reaccionado adaptándose a estas nuevas tendencias con el plan de estudios de Grado de Información y Documentación, diseñado teniendo como marco de referencia el Libro Blanco del año 2005 elaborado para el conjunto de universidades españolas que impartían estos estudios y que contó con la aportación de asociaciones y de empleadores.

El programa de estudios y la definición de las competencias del nuevo grado 
incluye como asignaturas obligatorias: Técnicas de comunicación, Fundamentos de la cognición humana, Información y sociedad, Análisis de la necesidad de información, Estadística aplicada, Aspectos legales de la información, Desarrollo de colecciones, Metodología de la búsqueda, Evaluación y calidad, Formación semántica, Márquetin... Estas nuevas materias alejan a los futuros profesionales del mundo de la literatura i los sitúa como prescriptores de la información al tiempo que los introducen en el mundo de la gestión.

La propuesta universitaria reconoce que, aunque los profesionales bibliotecarios, una vez formados, pueden utilizar técnicas e instrumentos distintos, en función de donde desempeñen su actividad profesional (bibliotecas generales, especializadas, archivos, empresas...), disponen de un perfil profesional único para poder adaptarse a cualquier demanda laboral. Esta circunstancia, como nos indican Frías y Villalba (2009), en estudios sobre inserción laboral de los titulados, muestra que las bibliotecas generales, especializadas o centros de documentación continúan absorbiendo un porcentaje importante de titulados, pero también pone en evidencia que cada vez son más los graduados que encuentran trabajo en archivos, y que la demanda de un profesional polivalente con conocimientos de biblioteconomía y gestión de la documentación va en aumento.

\section{Más formación continuada. Competencias y habilidades}

Cuando parece que los caminos que siguen la formación universitaria y las necesidades del mercado laboral no terminan de converger, ni de convencer a algunos profesionales del sector, la formación continuada parece la única salida para estos técnicos que aún defienden que su perfil no necesita un cambio substancial.

Está formación no ha de limitarse únicamente a los bibliotecarios, sino que ha de incluir a todo el personal de la biblioteca: graduados y auxiliares de apoyo, a menudo poco valorados. Los datos son contundentes: de los mil dos cientos trabajadores de las bibliotecas públicas de la Diputación de Barcelona más de setecientos son auxiliares, los cuales no disponen hasta ahora de estudios específicos. Su formación se remite a cursos y cursillos que organizan las diferentes instituciones del sector y que, en el caso de Cataluña, son los diseñados por el Colegio de Bibliotecarios, Escuelas de verano y de invierno, organizadas por la Facultad de Biblioteconomía de la Universidad de Barcelona, la Generalitat de Catalunya y la Diputación de Barcelona.

Por otro lado, la oferta formativa universitaria de master y posgrados parece ser una importante vía para la formación especializada de titulados. Seguramente poder seguir la evolución de esta oferta nos indicará, en el futuro, las competencias requeridas por los empleadores. En este sentido hay que destacar la amplia oferta por parte de las universidades españolas de postgrados profesionales enfocados a los sistemas y servicios de información digital.

La opinión sobre la necesidad y la falta de formación es compartida y debatida por algunos expertos como se pone de manifiesto en el articulo: els bibliotecaris $i$ documentalistes estan capacitats per cobrir els llocs de treball emergents que estan sorgint?... 
(Andreu et al., 2010) del cual destacamos las siguientes opiniones por estar directamente relacionadas con el funcionamiento de la biblioteca pública.

Jordi Permanyer² opina que: "En las bibliotecas públicas realmente no encuentras a faltar competencias, sino que tengan habilidades en muchas cosas". "Cada equipamiento es municipal y aislado de los otros, por lo tanto el gestor ha de ser capaz de resolver cuestiones muy diversas. Los municipios pequeños tienen una realidad diferente de los municipios grandes y por lo tanto, hace falta implicación y resoluciones diferentes. Por ejemplo, en los municipios pequeños, los perfiles han de ser polivalentes y en los grandes se especializan más. En los municipios grandes los problemas aparecen en las direcciones y responsabilidades".

"Hace falta dotar a los profesionales de formación para que puedan asumir roles directivos, que es el déficit más grande. Más habilidades de gestión en todas sus facetas. El trabajo de un responsable de biblioteca es interdisciplinario con otros profesionales del municipio y tiene que tener habilidad para está forma de trabajar. Los responsables también han de saber posicionar aquella biblioteca o equipamiento dentro del municipio, esto quiere decir márquetin, cursos de directores, con el objetivo de perfeccionar los planes de actuación, las memorias, las gestiones con el concejal correspondiente, etc.”.

Amadeu Pons 3 sostiene: "Tenemos unas competencias transversales, por ejemplo el manejo de las tecnologías de la información, la capacidad de trabajo en equipo...; esto es fácil de enseñar. Pero hay competencias más difíciles de transmitir. Por ejemplo, la capacidad creativa y emprendedora”.

Por su parte Mónica Montero*4 considera: "Pero las competencias se enseñan. Las habilidades en comunicación se pueden ensayar. Se puede hacer mejor o peor, pero te han de enseñar a planificar y hacer un presupuesto. Un profesional, cuando sale de la carrera, lo ha de saber hacer".

De acuerdo con Jordi Permanyer, podemos asegurar que a los profesionales destinados a ocupar puestos de responsabilidad no les deberían faltar habilidades en el ámbito de la dirección y gestión, así como el establecimiento de sinergias con el ámbito político Sin embargo, también tenemos que considerar que estas habilidades son fundamentales en la formación del directivo pero que estos también han de ser creativos y emprendedores. En este sentido, Amadeu Pons señala que estas competencias no se pueden enseñar en las aulas.

Por otro lado, desde la empresa pública, Mónica Montero, también manifiesta la falta de algunas competencias entre los titulados al acabar sus estudios y asegura que el saber hacer se puede aprender, pero no deben faltar los conocimientos en gestión presupuestaria.

Del mismo modo y sin perder de vista las aportaciones anteriores, no podemos olvidar que los bibliotecarios, como profesionales de la información, trabajan directamente con las necesidades de los usuarios; por tanto, sus capacidades han de 
estar enfocadas hacia estas necesidades, que pueden ser simples o complejas dependiendo de la demanda. En ambos casos, los bibliotecarios han de poder y saber adaptarse a los cambios. La orientación y la formación son pilares necesarios para adquirir más herramientas, habilidades y capacidades para desarrollar su carrera profesional. Hay que señalar que las habilidades más importantes a desarrollar son las orientadas a la comunicación con los usuarios, con los colegas de profesión y con los superiores.

\section{Conclusiones}

Como sostienen Bailac y Hernández (2011) en su artículo Recortes y oportunidades, los cambios electorales que se acaban de producir en este año 2011 parece que conducirán a la definición de políticas públicas, que seguramente afectaran a la biblioteca pública. Los bibliotecarios tendrán que saber asumir la responsabilidad de defender y reforzar la posición de la biblioteca pública ante los cambios políticos y el nuevo momento económico. Esta perspectiva dibuja la trayectoria de futuro de la biblioteca pública y pide una nueva redefinición, no solamente exigida por las transformaciones que el desarrollo tecnológico está produciendo, sino también en la manera en que se genera, accede y se utiliza la información y el conocimiento.

Queda mucho por hacer, es preciso abandonar el conformismo que pueda albergar el personal bibliotecario y que impera ante el cambio informacional, que reclama la nueva realidad social en nuestras bibliotecas; unas instituciones donde parece dominar la hipervaloración de la tecnología por encima de los requerimientos de información reales del usuario. Los profesionales de nuestras bibliotecas deberán de modificar su perfil y consolidarse como prescriptores de la información pero, lo tendrán que hacer con carácter híbrido, no estrictamente documental.

La comunicación con el usuario se convierte en una pieza clave que conviene tener presente ya que es el principal factor de visibilidad de la biblioteca de cara a sus usuarios. Por este motivo es importante analizar los sistemas que utilizamos, el uso que se hace de ellos, la satisfacción del usuario y la calidad del servicio.

La biblioteca del futuro más inmediato ha de estar orientada al usuario y por ello debemos prepararnos para mejorar las técnicas comunicativas, pero también para mostrar a los usuarios el camino a seguir para conseguir un buen uso de nuestras instalaciones.

El papel de formadores en información es fundamental en un entorno donde impera la sobrevaloración de las competencias informáticas, por encima de las competencias relacionadas con la información, lo cual desequilibra la capacidad ciudadana en la sociedad digital. Un reciente estudio realizado por la British Library (2011), "Information behaviour of the researcher of the future" determina que hay algunos mitos con relación a las nuevas prácticas informacionales de la llamada generación digital (o Net Gen), generación Google (g-Google) o nativos digitales, entre otras denominaciones. Sus componentes, parecen no tener una idea clara de cuales son sus necesidades de información, lo que les impide orientar con éxito sus 
estrategias de búsqueda. Tampoco dedican apenas tiempo a evaluar y a contrastar la fiabilidad de las informaciones localizadas. Este hecho nos induce a pensar que disponer de capacidades tecnológicas en la búsqueda de información no los hace más competentes informacionalmente. $\mathrm{Y}$ en este escenario, las bibliotecas como unidades de información y agentes educativos, y a través de sus profesionales tienen un papel relevante y estratégico para formar usuarios en el uso autónomo, crítico y ético de la información.

Delante del espejismo tecnológico que hace creer que "todo" se encuentra en Internet o en Google (googlelización), cosa que hace superfluas las instituciones dedicadas a contener o transferir información, la biblioteca pública ha de actuar en la desintermediación del acceso a la información.

La formación deficiente, desfasada o inadecuada al mercado y a la demanda de las bibliotecas sólo se podrá paliar, de momento, con la requerida formación continuada, pero no únicamente dirigida a los bibliotecarios, sino también, como ya se ha dicho al personal auxiliar y de refuerzo.

Para el futuro más inmediato, podemos prever que los recursos y servicios en línea, la inclusión de elementos de la web social como los catálogos participativos, la presencia de redes, los servicios para dispositivos móviles y la descarga de libros electrónicos, irán adquiriendo protagonismo en las bibliotecas, pero de momento aún convivirán con espacios, servicios y recursos presénciales.

Es importante destacar que los profesionales de la biblioteca pública aunque muestren un elevado nivel de satisfacción hacia su profesión, consideran como escaso su reconocimiento social, al mismo tiempo que se sienten poco representados por los colegios profesionales. Hace falta pues, unificar los esfuerzos y las voces de los profesionales y de los colegios que los representan para hacerse escuchar delante de unas instituciones que solamente hablan de recortes y que pueden, de un plumazo, llevarse por delante la tarea realizada hasta ahora por bibliotecas y bibliotecarios.

En los últimos años, las actuaciones de fomento de la lectura y la construcción de nuevas bibliotecas han sido prioritarias en las políticas gubernamentales de nuestro país. Actualmente, el mundo de la cultura se ve amenazado por los anunciados recortes que pueden frustrar avances en materia de política bibliotecaria y de la información, una amenaza capaz de lanzar por la borda lo conseguido hasta ahora. Así pues, a los bibliotecarios les ha llegado el momento de actuar, es mucho lo que hay en juego y solo con la reflexión, la implicación y el compromiso podrán tirar adelante. Se juegan el futuro de la profesión.

\section{Notas}

1. El documento se presentó en el Consell de biblioteques celebrado en Barcelona el 17 de septiembre de 2009.

2. Gerente del Servei de Biblioteques de la Xarxa de Biblioteques Municipals de la Diputació de Barcelona. Actualmente la Red está formada por 200 bibliotecas y 9 bibliobuses.

3. Profesor de la Facultad de Biblioteconimía y Documentación de la Universidad de Barcelona. 
4. Responsable de Gestión de la Información en Biocat.

\section{Bibliografia}

Agencia Nacional de Evaluación de la Calidad y Acreditación (ANECA) (2004). Título del grado en Información y Documentación. Disponible en: <http://www.aneca.es/var/media/150424/ libroblanco_jun05_documentacion.pdf> [Consulta: 13 Julio 2011].

Andreu, M., Domínguez, C., Montero, M., Permanyer, J., Pons, A. y Díaz, A. (2010) “Els bibliotecaris i documentalistes estan capacitats per cobrir els llocs de treball emergents que estan sorgint? La relació entre l'oferta i la demanda és bona? Quins perfils cerquen les empreses o administracions públiques? Quins perfils troben?”, Item, No 53, pp. 10-18.

Atenas, J. (2010) “Bibliotecaris, documentalistes i competències professionals”, Item, No 53, pp. 20-29.

Bailac, A. y Hernandez H. (2011) "Recortes y oportunidades: Las bibliotecas y sus profesionales ante unas elecciones en época de crisis”, Mi biblioteca, No 22, pp. 30-36.

British Library (2011), Information behaviour of the researcher of the future. Disponible en: <http:// www.jisc.ac.uk/media/documents/programmes/reppres/ggworkpackageii.pdf> [Consulta: 13 Julio 2011].

Col.legi Oficial de Bibliotecaris i Documentalistes de Catalunya (2011) "Per la dignificació de la professió: una reflexió", Butlletí del Col.legi Oficial de Bibliotecaris i Documentalistes de Catalunya, No 195. Disponible en: <http://www.cobdc.net/document-195/2011/04/per-la-dignificacio-de-laprofessio-una-reflexio/> [Consulta: 13 Julio 2011].

Durban-Roca, G., Serrat-Brustenga, M. y Sunyer-Lázaro, S. (2010) "Els nadius digitals i ALFIN: un repte per als diferents entorns bibliotecaris a l'Estat espanyol?”, Item, No 50, pp. 31-45.

Frias, J. A. y Villalba del Monte, R. (2009), "De la Biblioteconomía a la Información: La formación universitaria de los bibliotecarios españoles en el Espacio Europeo de Educación Superior", comunicación presentada en XV Jornadas bibliotecarias de Andalucía, Bibliotecas: rompiendo barreras, tejiendo redes. 15-17 Octubre, Córdoba.

García Sáenz de Tejada, C., Andreu Felipe, I. y Alcázar Ruiz, C. D. (2009), "La información es poder.... si sabes usarla. Campaña de promoción del profesional de la documentación y la información. Experiencias", comunicación presentada en XI Jornadas Españolas de Documentación. 20-22 Mayo, Zaragoza.

Generalitat de Catalunya (2009) Imagina la biblioteca pública del S. XXI: extracció de dades de les enquestes. Disponible en: <http://cultura.gencat.cat/redir.asp?codipag=161> [Consulta: 13 Julio 2011].

Gómez Hernández, J. A., Hernández Sánchez, H. y Merlo Vega, J. A. (2011) "Prospectiva de una profesión en constante evolución. Estudio FESABID sobre los profesionales de la información", comunicación presentada en XII Jornadas Españolas de Documentación EBLIDA-NAPLE Conference. 25-27 Mayo, Málaga. Disponible en: <http://www.fesabid.org/malaga2011/prospectiva-de-unaprofesion-en-constante-evolucion> [Consulta: 13 Julio 2011].

Hernández, H. (2010) Inmigrantes, lectura y biblioteca pública. Disponible en: <http:// www.bibliotecaspublicas.info/hhs/inmigrantes_lectura_yBP.pdf> [Consulta: 13 Julio 2011 ].

Lozano Díaz, R. (2006) La biblioteca pública del siglo XXI: atendiendo clientes, movilizando personas, Trea, Gijón. 